\title{
Effective interpretations of a diphoton excess
}

\author{
Laure Berthier, ${ }^{a}$ James M. Cline, ${ }^{a, b}$ William Shepherd ${ }^{a}$ and Michael $\operatorname{Trott}^{a}$ \\ ${ }^{a}$ Niels Bohr International Academy 8 Discovery Center, \\ Niels Bohr Institute, University of Copenhagen, \\ Blegdamsvej 17, DK-2100, Copenhagen, Denmark \\ ${ }^{b}$ Department of Physics, McGill University, \\ 3600 Rue University, Montréal, Québec, Canada H3A $2 T 8$ \\ E-mail: berthier@nbi.ku.dk, jcline@physics.mcgill.ca, \\ shepherd@nbi.ku.dk, michael.trott@cern.ch
}

ABStRact: We discuss some consistency tests that must be passed for a successful explanation of a diphoton excess at larger mass scales, generated by a scalar or pseudoscalar state, possibly of a composite nature, decaying to two photons. Scalar states at mass scales above the electroweak scale decaying significantly into photon final states generically lead to modifications of Standard Model Higgs phenomenology. We characterise this effect using the formalism of Effective Field Theory (EFT) and study the modification of the effective couplings to photons and gluons of the Higgs. The modification of Higgs phenomenology comes about in a variety of ways. For scalar $0^{+}$states, a component of the Higgs and the heavy boson can mix. Lower energy phenomenology gives a limit on the mixing angle, which gets generated at one loop in any theory explaining the diphoton excess. Even if the mixing angle is set to zero, we demonstrate that a relation exists between lower energy Higgs data and a massive scalar decaying to diphoton final states. If the new boson is a pseudoscalar, we note that if it is composite, it is generic to have an excited scalar partner that can mix with a component of the Higgs, which has a stronger coupling to photons. In the case of a pseudoscalar, we also characterize how lower energy Higgs phenomenology is directly modified using EFT, even without assuming a scalar partner of the pseudoscalar state. We find that naturalness concerns can be accommodated, and that pseudoscalar models are more protected from lower energy constraints.

KEYworDS: Beyond Standard Model, Effective field theories

ARXIV EPRINT: 1512.06799 


\section{Contents}

1 Introduction $\quad 1$

1.1 Properties of the diphoton excess at $\sim 750 \mathrm{GeV} \quad 2$

2 Scalar models $\quad 3$

2.1 Integrating out $S \quad 4$

$\begin{array}{lll}2.2 & \text { Generating the } S H^{\dagger} H \text { operator } & 7\end{array}$

2.3 Direct matching contributions to the $O_{H B}, O_{H G}$ operators 8

2.4 Constraints from Electroweak Precision Data 8

2.5 Constraints from run I Higgs data 9

$\begin{array}{lll}2.5 .1 & \text { Mixing domination } & 10\end{array}$

2.5.2 Reproducing the width 11

2.5.3 Matching domination 11

3 Consistency of pseudoscalar models with lower energy data 12

4 Conclusions $r$

$\begin{array}{lr}\text { A One-loop results } & 14\end{array}$

\section{Introduction}

The global data set reported by LEP, the Tevatron, LHC and a host of low-energy experiments is consistent with the Standard Model (SM) of particle physics. With the discovery of a $0^{+}$scalar $(h)$ consistent in its properties with the scalar $0^{+}$component of the SM Higgs doublet $(H)$, any extension of the SM that aims to explain new phenomena is constrained by an even larger bevy of lower energy tests. With the initial reporting of run II data at $\sqrt{s} \cong 13 \mathrm{TeV}$, lower energy tests now include the properties of the "Higgs pole" measurements, fixed to $m_{h} \cong 125 \mathrm{GeV}$, measured at $\sqrt{s} \cong 7,8 \mathrm{TeV}$ in run I. In this paper, we discuss a set of consistency conditions for scalars with mass scales $m_{s} \gg m_{h}$ that generate a significant decay to diphoton final states, arising from these lower energy measurements. We will assume that the $125 \mathrm{GeV}$ scalar is approximately the SM Higgs boson and study the perturbation of its properties using the Standard Model Effective Field Theory (SMEFT) formalism. The modification of the SM Higgs properties comes about in a variety of ways. For example, a component of the SM Higgs can mix with a new $0^{+}$scalar or higher-mass resonances. The constraints we derive on the mixing from the experimentally established Higgs couplings must be respected by any models with new scalars that can mix significantly with the Higgs. Other constraints, not directly tied to mixing, are also present when studying low-energy phenomenology. We characterize these matching effects using the SMEFT. 
Our motivation is the report of a slight excess of diphoton events in the ATLAS and CMS data $[1,2]$ at $\sim 750 \mathrm{GeV}$. This excess might be, and arguably most likely is, a statistical fluctuation [1-3]. ${ }^{1}$ However, the possibility that this excess is generated by new physics has received a lot of attention. Many authors have considered models in which the hypothesized scalar is composite, due to the need for it to couple to gluons or photons despite it being neutral. It is interesting to consider what effects such a state could have on the observed properties of the Higgs boson. Mixing of $h$ with any new states that decay to diphotons will introduce a shift in the expected branching ratio for $h \rightarrow \gamma \gamma$. With the measurements of run I, it is known that any such perturbation cannot greatly alter the observed branching ratio, which is $B(h \rightarrow \gamma \gamma) \cong 2 \times 10^{-3}$. Numerous higher dimensional operators at lower scales have also been probed at LHC in run I, and in Electroweak Precision Data (EWPD) studies. We study the consequences of a diphoton excess at $\sim 750 \mathrm{GeV}$ in a wide class of models arising from consistency with these lower energy tests.

The purpose of this paper is to further develop these consistency tests and to apply them to generic models that could explain the putative excess. Although some of the constraints we will derive can be satisfied by choosing parameters such that the scalar-h mixing angle is sufficiently small in some models, it is interesting to ask whether such values are natural or if they require fine tuning. This issue is sharpened by the fact that the mixing of interest is necessarily generated by the same operators that are assumed to exist for the purpose of explaining the diphoton events. Other (weaker) constraints we derive are not related to the scalar-h mixing angle at all, but still must be respected.

On the other hand, pseudoscalar states are forbidden by parity from mixing with $h$. However, we will argue that pseudoscalar states in the spectrum of a strongly confining sector are likely to be accompanied by scalar states, with an even stronger (effective) coupling to photons, on fairly general grounds, leading to indirect constraints on sectors with composite pseudoscalars as the lightest states. Further, we characterize how pseudoscalar states still lead to modified properties of the SM Higgs in lower energy experiments using the formalism of the SMEFT. The conditions we develop provide a challenge to the construction of consistent strongly interacting models for the diphoton excess.

\subsection{Properties of the diphoton excess at $\sim 750 \mathrm{GeV}$}

The properties of the $\sim 750 \mathrm{GeV}$ diphoton excess have been reported in detail by the experimental collaborations $[1,2]$. In brief summary, the excess at $\sim 750 \mathrm{GeV}$ in diphoton final states is characterised as resonant production with an approximate cross section $\sigma(p p \rightarrow S \rightarrow \gamma \gamma) \approx 8 f b$. The excess in ATLAS data has a local statistical significance of $3.6 \sigma$ and global significance of $2.0 \sigma$, while that of CMS is at $2.6 \sigma$, with a global significance of only $1.2 \sigma$. The two experiments have differing preferences for the width of the resonance, with CMS preferring a narrow state relative to the experimental resolution of about $6 \mathrm{GeV}$, while the ATLAS data prefer a larger width of around $45 \mathrm{GeV}$. The width

\footnotetext{
${ }^{1}$ We note that the arguments we advance are quite general for higher scale composite resonances that have a significant branching fraction to diphoton final states, even if the current excess is a statistical fluctuation.
} 
preferences in both cases are clearly very weak and there is no joint fit specifying a width preference reported by the experimental collaborations. For this reason we will formulate our consistency conditions in a manner that allows the width to be easily adjusted, to a future experimental value, that is more consistent between the experimental results.

\section{Scalar models}

The Landau-Yang theorem $[4,5]$ states that a resonance decaying to diphotons can only have spin 0 or spin 2 . Here we do not consider spin 2 models, or the simultaneous production of other, undetected, states to consider other possibilities. Spin zero particles can be either scalar or pseudoscalar, and either fundamental or composite. We first focus on the scalar case.

In a fairly general class of models, the scalar field $S$ couples to gluons, photons and possibly quarks in order to explain the production and decay of $S$ into photons that give the diphoton excess. For a scalar of mass $m_{s}$ and width $\Gamma_{s}$, one can express the extra (due to $S$ ) contribution to the cross section times branching ratio to photons as

$$
\begin{aligned}
\Delta \sigma(p p \rightarrow S \rightarrow \gamma \gamma) & =\frac{\Gamma(S \rightarrow \gamma \gamma)}{m_{s} \Gamma_{s} s}\left[\mathcal{C}_{g g} \Gamma(S \rightarrow g g)+\mathcal{C}_{\gamma \gamma} \Gamma(S \rightarrow \gamma \gamma)+\sum_{q} \mathcal{C}_{q} \Gamma(S \rightarrow q \bar{q})\right] \\
& =2.3 \mathrm{pb} \times \frac{\Gamma(S \rightarrow \gamma \gamma)}{m_{s}} \sum_{i} \mathcal{C}_{i} \operatorname{Br}(S \rightarrow i i)
\end{aligned}
$$

For $\sqrt{s}=13 \mathrm{TeV}$ the dimensionless coefficients are approximately

$$
\mathcal{C}_{i}=\left\{\mathcal{C}_{\gamma \gamma}, \mathcal{C}_{b}, \mathcal{C}_{c}, \mathcal{C}_{s}, \mathcal{C}_{u}, \mathcal{C}_{d}, \mathcal{C}_{g g}\right\} \simeq\{78.3,15.3,35.7,83,1054,627,2137\}
$$

For example if $\Gamma(s \rightarrow \gamma \gamma) \sim \Gamma(s \rightarrow g g) \sim 0.01 m_{s}$, we find $\sigma \sim f b \times \mathcal{C}_{i}$. The $\mathcal{C}_{\gamma \gamma}$ term was reported in [6] to be 0.53 using the equivalent photon approximation, assuming that the inverse of the impact parameter scaled to the proton radius is $r_{\star} \sim 0.13$. This result is similar to the elastic scattering result reported in ref. [7]. Inelastic scattering results are dominant for this production mechanism [7]. Recent estimates of the combined inelasticinelastic, elastic-inelastic and elastic-elastic photoproduction [7-9] give a corrected $\mathcal{C}_{\gamma \gamma}=$ 78.3. The latter coefficients were generated in ref. [10] at a renormalization scale $\mu=m_{s}$ using MSTW2008 parton distribution functions (PDFs) [11]. The parton luminosities are such that gluonic or photonic production of the state can dominate. Utilizing the quark production mechanism has been examined in ref. [12], and found to be challenging.

We focus on the cases of production and decay through $g g \rightarrow S$ and $S \rightarrow \gamma \gamma$. We consider the case where the scalar field $S$ couples via the operators ${ }^{2}$

$$
\mathcal{L}_{\text {int }}=\frac{c_{G} g_{3}^{2}}{\Lambda_{g}} S G^{\mu \nu} G_{\mu \nu}+\frac{c_{B} g_{1}^{2}}{\Lambda_{\gamma}} S B^{\mu \nu} B_{\mu \nu}+\frac{c_{W} g_{2}^{2}}{\Lambda_{\gamma, 2}} S W^{\mu \nu} W_{\mu \nu}
$$

\footnotetext{
${ }^{2}$ Of course in a general scalar singlet case, all dimension five operators of the form $S \times \mathcal{L}_{S M}$ are present. And considering dimension six operators many other operators, for example, $S^{2} B^{\mu \mu} B_{\mu \nu}$ are also present. Our purpose is to link a high energy diphoton excess in a minimal scenario with lower energy phenomenology, so these further Lagrangian terms with unknown Wilson coefficients are neglected.
} 
Note that some notation is reused here from the SMEFT operator basis. Here $g_{1,2,3}$ refer respectively to the $B, W$ and $G$ gauge couplings. Decays through the latter operator lead to enhanced couplings to $W^{+} W^{-}, Z Z$ and $Z \gamma$ final states, while the first two yield smaller levels of such decays. Decays to $Z \gamma$ are disfavoured by correlated searches at the mass scale $\sim 750 \mathrm{GeV}$. Ref [13] reports a 95\% C.L. bound on $\sigma\left(p p \rightarrow S \rightarrow Z\left(\ell^{+} \ell^{-}\right) \gamma\right)$ of $\lesssim 0.3 \mathrm{fb}$, whereas the expected deviation associated with the $\gamma \gamma$ excess, assuming the decay is generated by the coupling to $\mathrm{SU}(2)_{\mathrm{L}}$ (and the $Z$ and decaying to $\ell^{+} \ell^{-}$), is $\sim 2 \mathrm{fb} .{ }^{3}$ It is possible to cancel away this tension by having both the operators $O_{B}$ and $O_{W}$ present with correlated Wilson coefficients [14] (implicitly defined in eq. (2.3)); however we will not further consider generating $c_{W}$ since $Z \gamma$ bounds are not the essential point of this study. We only consider models with negligible $c_{W}$. The decay widths are related to the remaining dimension-five operators, introduced with the given normalization, as

$$
\Gamma(S \rightarrow \gamma \gamma)=\frac{4 \pi \alpha_{e w}^{2} m_{s}^{3}}{\Lambda_{\gamma}^{2}} c_{B}^{2}, \quad \Gamma(S \rightarrow g g)=\frac{32 \pi \alpha_{s}^{2} m_{s}^{3}}{\Lambda_{g}^{2}} c_{G}^{2} .
$$

Using (2.4), we can rewrite the cross section for the diphoton excess (2.1) as

$$
\frac{\Delta \sigma(p p \rightarrow S \rightarrow \gamma \gamma)}{8 \mathrm{fb}}\left(\frac{\Gamma_{s}}{45 \mathrm{GeV}}\right) \cong 6546\left(\frac{m_{s}^{2} c_{B}^{2}}{\Lambda_{\gamma}^{2}}\right)\left[\left(\frac{m_{s}^{2} c_{G}^{2}}{\Lambda_{g}^{2}}\right)+3.5 \times 10^{-5}\left(\frac{m_{s}^{2} c_{B}^{2}}{\Lambda_{\gamma}^{2}}\right)\right]
$$

The gauge couplings $\alpha_{s}, \alpha_{e w}$ are evaluated at the scale $m_{s} \cong 750 \mathrm{GeV}$. We note that, in the presence of the operators generated by integrating out the scalar $S$ at its mass, the running of $\alpha_{s}, \alpha_{e w}$ is modified [15]. The corresponding Wilson coefficients in the SMEFT can receive contributions from other unknown UV physics. Such nonresonant contributions are neglected. We also note that the running effect on the production and decay of the scalar particle is higher order in the power counting, and neglected. We run $\alpha_{s}, \alpha_{e w}$ up from the scale $m_{Z}$ using SM relations, so that $\alpha_{s}(750 \mathrm{GeV}) \cong 0.09$, and $\alpha_{\text {ew }}(750 \mathrm{GeV}) \cong 1 / 126.5$.

In a valid EFT expansion, one expects that $m_{s}<\Lambda_{\gamma, g}$. If we normalized the Wilson coefficients proportional to a loop factor $\sim(16 \pi)^{-2}$ in the case of some weakly coupled renormalizable UV models, large Wilson coefficients are required. Extreme solutions where $c_{B} / \Lambda_{\gamma} \ll c_{G} / \Lambda_{g}$ or $c_{B} / \Lambda_{\gamma} \gg c_{G} / \Lambda_{g}$ are possible. One naturally expects $\Lambda_{g} \sim \Lambda_{\gamma}$ and $c_{B}$ and $c_{G}$ to differ only by group theory factors, in scenarios where a common mediator generates the two decays.

\section{$2.1 \quad$ Integrating out $S$}

Minimal scalar field models have the potential Lagrangian terms

$$
\begin{aligned}
\mathcal{L}_{V}= & -\lambda_{S M}\left(H^{\dagger} H-\frac{1}{2} v^{2}\right)^{2}-\frac{m_{s}^{2}}{2} S^{2}+\frac{\kappa}{4 !} S^{4}+\lambda \Lambda_{c} S H^{\dagger} H+\lambda_{2} \Lambda_{c} S^{3}+\lambda_{3} S^{2}\left(H^{\dagger} H\right) \\
& +\lambda_{4} \Lambda_{c}^{3} S+\cdots
\end{aligned}
$$

\footnotetext{
${ }^{3}$ Here we have used $\sigma(p p \rightarrow s)[8 \mathrm{TeV}] / \sigma(p p \rightarrow s)[13 \mathrm{TeV}] \cong 0.21$, assuming $g g$ production is dominant. Note that the excess is in $13 \mathrm{TeV}$ data while the bound in ref. [13] is for $8 \mathrm{TeV}$ data.
} 
No unbroken discrete symmetry exists that forbids the $\lambda, \lambda_{2}$ terms, since $S$ decays. There are no significant consequences for our analysis due to the $\lambda_{4} \Lambda_{c}^{3} S$ term but we note that this term is regenerated from the $S^{3}$ term in a tadpole diagram if this term was set to zero. Here $\Lambda_{c}$ is the cutoff scale of the toy model effective Lagrangian, and we assume that some unknown states with a mass scale $\sim \Lambda_{c}$ generate the coupling of $S$ to photons and gluons. Due to the presence of effective dimension five terms in the Lagrangian, higher order terms are also generated in the potential suppressed by $1 / \Lambda_{c}$. Since $m_{s} \gg v$, it is interesting to consider the case that $S$ decays through manifestly $\mathrm{SU}(3) \times \mathrm{SU}(2)_{\mathrm{L}} \times \mathrm{U}(1)_{\mathrm{Y}}$ invariant operators. Integrating out $S$ one obtains the effective lagrangian suitable for describing Higgs-gauge boson couplings,

$$
\mathcal{L}=\mathcal{L}_{S M}+\frac{C_{H G}\left(m_{s}\right) g_{3}^{2}}{\Lambda^{2}} H^{\dagger} H G^{\mu \nu} G_{\mu \nu}+\frac{C_{H B}\left(m_{s}\right) g_{1}^{2}}{\Lambda^{2}} H^{\dagger} H B^{\mu \nu} B_{\mu \nu}
$$

where

$$
\frac{C_{H G}\left(m_{s}\right)}{\Lambda^{2}}=\frac{c_{G} \lambda}{m_{s}^{2}} \frac{\Lambda_{c}}{\Lambda_{g}}, \quad \frac{C_{H B}\left(m_{s}\right)}{\Lambda^{2}}=\frac{c_{B} \lambda}{m_{s}^{2}} \frac{\Lambda_{c}}{\Lambda_{\gamma}} .
$$

At lower scales $\mu$, the Wilson coefficients are then, in a leading log approximation, (only retaining the Yukawa couplings $\left.Y_{t}, Y_{b}\right)[15-18]$

$$
\begin{aligned}
C_{H G}(\mu) & =\left(1-\log \left[\frac{m_{s}}{\mu}\right] \frac{12 \lambda+2 N_{c}\left(\left(\sqrt{2} M_{t}\right)^{2}+\left(\sqrt{2} M_{b}\right)^{2}\right) / v^{2}-6 g_{1}^{2} y_{h}^{2}-9 g_{2}^{2} / 2}{16 \pi^{2}}\right) C_{H G}\left(m_{s}\right), \\
C_{H B}(\mu) & =\left(1-\log \left[\frac{m_{s}}{\mu}\right] \frac{12 \lambda+2 N_{c}\left(\left(\sqrt{2} M_{t}\right)^{2}+\left(\sqrt{2} M_{b}\right)^{2}\right) / v^{2}+2 y_{h}^{2} g_{1}^{2}-9 g_{2}^{2} / 2}{16 \pi^{2}}\right) C_{H B}\left(m_{s}\right), \\
C_{u H}(\mu) & =-\frac{\sqrt{2} M_{t}}{v} \frac{2 g_{3}^{4}}{\pi^{2}} \log \left[\frac{m_{s}}{\mu}\right] C_{H G}\left(m_{s}\right)-\frac{\sqrt{2} M_{t}}{v} \frac{3\left(y_{h}^{2}+2 y_{q} y_{u}\right)}{4 \pi^{2}} g_{1}^{4} \log \left[\frac{m_{s}}{\mu}\right] C_{H B}\left(m_{s}\right), \\
C_{d H}(\mu) & =-\frac{\sqrt{2} M_{b}}{v} \frac{2 g_{3}^{4}}{\pi^{2}} \log \left[\frac{m_{s}}{\mu}\right] C_{H G}\left(m_{s}\right)-\frac{\sqrt{2} M_{b}}{v} \frac{3\left(y_{h}^{2}+2 y_{q} y_{d}\right)}{4 \pi^{2}} g_{1}^{4} \log \left[\frac{m_{s}}{\mu}\right] C_{H B}\left(m_{s}\right), \\
C_{u G}(\mu) & =\frac{\sqrt{2} M_{t}}{4 \pi^{2} v} g_{3}^{3} \log \left[\frac{m_{s}}{\mu}\right] C_{H G}\left(m_{s}\right), \\
C_{u B}(\mu) & =\frac{\sqrt{2} M_{t}}{16 \pi^{2} v}\left(2 g_{1}^{3}\left(y_{q}+y_{u}\right)\right) \log \left[\frac{m_{s}}{\mu}\right] C_{H B}\left(m_{s}\right), \\
C_{d G}(\mu) & =\frac{\sqrt{2} M_{b}}{4 \pi^{2} v} g_{3}^{3} \log \left[\frac{m_{s}}{\mu}\right] C_{H G}\left(m_{s}\right), \\
C_{d B}(\mu) & =\frac{\sqrt{2} M_{b}}{16 \pi^{2} v}\left(2 g_{1}^{3}\left(y_{q}+y_{d}\right)\right) \log \left[\frac{m_{s}}{\mu}\right] C_{H B}\left(m_{s}\right), \\
C_{H W B}(\mu) & =-\frac{4 g_{1}^{3} g_{2} y_{h}}{16 \pi^{2}} \log \left[\frac{m_{s}}{\mu}\right] C_{H B}\left(m_{s}\right),
\end{aligned}
$$

with $y_{h}=1 / 2, y_{q}=1 / 6, y_{u}=2 / 3, y_{d}=-1 / 3$ being the hypercharges of the indicated particles. Here the $O_{u H}, O_{d H}, O_{e H}, O_{u G}, O_{d G}, O_{u B}, O_{d B}, O_{H W B}, O_{H}$ operators are defined 
as in ref. [19]. The Higgs potential is also changed in a nontrivial fashion

$$
\begin{aligned}
\delta \lambda_{S M}(\mu)= & -\frac{3 m_{h}^{2}}{4 \pi^{2}} g_{1}^{4} y_{h}^{2} \log \left[\frac{m_{s}}{\mu}\right] C_{H B}\left(m_{s}\right), \\
C_{H}(\mu)= & -\frac{3}{\pi^{2}} \lambda\left(g_{1}^{4} y_{h}^{2}\right) \log \left[\frac{m_{s}}{\mu}\right] C_{H B}\left(m_{s}\right) \\
& +\left(\frac{3}{\pi^{2}} g_{1}^{6} y_{h}^{4}+\frac{3}{4 \pi^{2}} g_{1}^{4} g_{2}^{2} y_{h}^{2}\right) \log \left[\frac{m_{s}}{\mu}\right] C_{H B}\left(m_{s}\right) .
\end{aligned}
$$

Here $\delta \lambda_{S M}$ is the modification of the running of the Higgs self-coupling relative to how it runs in the SM, below the scale $m_{s}$. It is interesting to note that these one-loop effects would have a nontrivial implication for the running and shape of the potential, if the diphoton excess was substantiated. We will resist drawing conclusions about the fate of the universe due to this observation. The modified potential will redefine the effective Higgs vacuum expectation value, but in an unobservable fashion in current experiments. For the particular case of Higgs physics, to get a sense of the impact on lower energy phenomenology, we note

$$
\begin{aligned}
C_{H G}\left(m_{h}\right) & \cong 0.94 C_{H G}\left(m_{s}\right), \\
C_{H B}\left(m_{h}\right) & \cong 0.94 C_{H B}\left(m_{s}\right), \\
C_{u H}\left(m_{h}\right) & \cong-0.46 C_{H G}\left(m_{s}\right)-0.0011 C_{H B}\left(m_{s}\right), \\
C_{d H}\left(m_{h}\right) & \cong-0.011 C_{H G}\left(m_{s}\right)-7.85 \times 10^{-6} C_{H B}\left(m_{s}\right), \\
C_{u G}\left(m_{h}\right) & \cong 0.054 C_{H G}\left(m_{s}\right), \\
C_{d G}\left(m_{h}\right) & \cong 0.0013 C_{H G}\left(m_{s}\right) \\
C_{u B}\left(m_{h}\right) & \cong 8.8 \times 10^{-4} C_{H B}\left(m_{s}\right), \\
C_{d B}\left(m_{h}\right) & \cong-4.4 \times 10^{-6} C_{H B}\left(m_{s}\right) \\
C_{H W B}\left(m_{h}\right) & \cong-6.9 \times 10^{-4} C_{H B}\left(m_{s}\right)
\end{aligned}
$$

Flavour indices have been suppressed, due to the scenario considered. There is a (up-down quark) flavour non-universal effect. Note the large effect on the top Yukawa coupling at the low scale. The (assumed) SM Higgs field at $125 \mathrm{GeV}$ coupling to the top gets modified as

$$
\kappa_{t}-1=\frac{v^{2}}{\sqrt{2} \Lambda^{2}}\left(0.46 C_{H G}\left(m_{s}\right)+0.0011 C_{H B}\left(m_{s}\right)\right) .
$$

Similarly one finds a modification for the coupling of the $h Z Z$ interaction of the form

$$
\left(0.94 s_{\theta}^{2}\right) \sqrt{2} v h Z^{\mu \nu} Z_{\mu \nu} C_{H B}\left(m_{s}\right)
$$

with $s_{\theta}^{2}$ referring to the Weinberg angle. The correction to the angle due to $C_{H W B}$ for this term is higher order. This correction leads to an effective modification of $\kappa_{Z}$. Taking into account the typical offshellness in the decays $h \rightarrow Z Z^{\star}$ into fermion final states [20], one finds

$$
\kappa_{Z}-1 \cong 0.2 \frac{m_{w}^{2}}{\Lambda^{2}} C_{H B}\left(m_{s}\right) .
$$


The effects on $\kappa_{Z}, \kappa_{t}$ are in general subdominant in the minimal scenario considered and can be neglected. In general, a scalar singlet of the form considered above is well isolated from inducing large low-energy effects.

As states of mass scale $\Lambda_{c}$ generate the $c_{B}$ operator at the scale $\Lambda_{\gamma}$, it is necessary that they are charged under $\mathrm{U}(1)_{Y}$. One expects a large number of operators to be generated at the scale $\Lambda_{c}$, with contributions to operators that include SM states that are charged under $\mathrm{U}(1)_{Y}$, at least at the two-loop level. In this case, the detailed impact on low-energy phenomenology can differ from the minimal case sketched here. When the Wilson coefficient of $S H^{\dagger} H$ is suppressed, two-loop effects can be comparable, or dominant, over the effects that we study in detail. One also expects one-loop contributions to the operators $O_{H G}, O_{H B}$ on general grounds. Our analysis assumes that such direct matching contributions are small enough to be neglected. The couplings of the states that generate $C_{G, B}$ (for $S$ in eq. (2.3)) to the SM Higgs are unknown and can be small.

\subsection{Generating the $S H^{\dagger} H$ operator}

On general grounds one expects the coupling of the scalar state to be sizable with $H^{\dagger} H$, and for the scalar to have a sizable self-coupling term $S^{3}$. These operators are relevant. Of course, pure naturalness expectations for scalar sectors are under pressure due to the measured Higgs mass. Assuming $\lambda \Lambda_{c} \sim[\mathrm{TeV}]$ the induced Higgs mass value is not strongly perturbed as $\Delta m_{h}^{2} \sim[\mathrm{TeV}]^{2} / 8 \pi^{2}$, so considering separations of scales where $\lambda \Lambda_{c}>m_{s}$ by an order one factor does not introduce significant extra tuning to the Higgs sector.

As we will characterize in more detail below, the scenario where $\lambda \Lambda_{c}$ is a value proximate to the cutoff scale, is problematic. ${ }^{4}$ At scales below $m_{s}$, the presence of the $S H^{\dagger} H$ operator leads to the higher dimensional operators $O_{H B}, O_{H G}$ in the SMEFT. If it is assumed that $\lambda \lambda_{c}$ is somehow suppressed, or fixed to zero, quantum corrections regenerate this mixing due to the interactions assumed above, to explain the excess in eq. (2.5). The mixing between $S$ and $h$ due to $c_{B}$ is generated by the one-loop diagram shown in figure 1(a). This gives a Naive Dimensional Analysis (NDA) [22] estimate of the coefficient of $S H^{\dagger} H$ of the form

$$
\lambda \Lambda_{c} \gtrsim \frac{g_{1}^{4} m_{s}^{2}}{32 \pi^{2}} \frac{c_{B}\left(m_{s}\right)}{\Lambda_{\gamma}},
$$

when the bare and loop induced terms of the Wilson coefficient of the operator $S H^{\dagger} H$ are not canceled against one another, at the scale $\mu=m_{s}$. The generation of $S H^{\dagger} H$ due to $c_{G}$ is a two-loop effect. A typical diagram is given in figure 1(b). The divergence in the diagram leading to the mixing is approximately

$$
\sim \operatorname{Tr}\left[T_{A} T_{A}\right] y_{t}^{2} g_{3}^{2} \frac{m_{s}^{2} c_{G}}{\left(16 \pi^{2}\right)^{2} \epsilon \Lambda_{g}},
$$

in dimensional regularization in $d=4-2 \epsilon$ dimensions. The colour factors enhance the magnitude of the diagram, as expected. ${ }^{5}$ The $\epsilon$ poles cancel in the matching onto the

\footnotetext{
${ }^{4}$ This observation was also pointed out while this draft was being finalized in ref. [21].

${ }^{5}$ Note that this is only a single pole divergence, despite being a two-loop graph. This is because the subgraph coupling $H^{\dagger} H$ to two gluons is finite in the SM.
} 


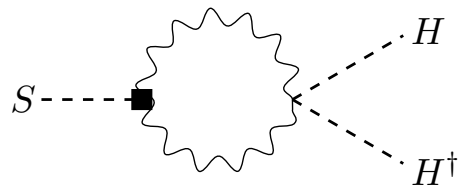

(a)

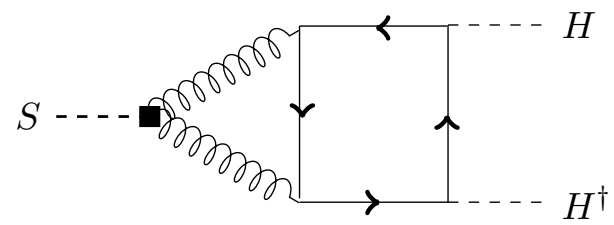

(b)

Figure 1. Diagrams generating the mixing of $S$ and $H$ at one loop due to the couplings required for $g g \rightarrow S$ and $S \rightarrow \gamma \gamma$, illustrated with the insertion of a box.

lower energy theory. However, we utilize the corresponding finite terms generated from the logarithmic dependence linked to the divergence in this diagram as an NDA-inspired estimate of the size of the Wilson coefficient of the $S H^{\dagger} H$ operator.

\subsection{Direct matching contributions to the $O_{H B}, O_{H G}$ operators}

There are also other direct one-loop contributions to the Wilson coefficients of the SMEFT operators shown in figure 2(a,b). Figure 2(a) depends on an unknown scalar coupling in the potential $-\lambda_{3}$, but does not require the operator $S H^{\dagger} H$ to generate an effective low scale $C_{H B}, C_{H G}$. Consider calculating figure $2(\mathrm{a})$ in dimensional regularization. The matching coefficient onto the SMEFT then receives a contribution from the finite parts of the on shell diagrams in the full and effective theories (in this case the SMEFT), while dropping the $1 / \epsilon$ poles. ${ }^{6}$ We have calculated the diagrams in figure 2 (see the appendix); a simple 1-loop estimate of the NDA minimum for the SMEFT operator's Wilson coefficients is adequate for our bounds. We require that

$$
\begin{aligned}
& \left|\frac{C_{H B}}{\Lambda^{2}}\right| \gtrsim \frac{\left(\lambda_{3}+y_{h}^{2} g_{1}^{2}\right) g_{1}^{2} c_{B}^{2}}{4 \pi^{2} \Lambda_{\gamma}^{2}}, \\
& \left|\frac{C_{H G}}{\Lambda^{2}}\right| \gtrsim \frac{\lambda_{3} g_{3}^{2} c_{G}^{2}}{4 \pi^{2} \Lambda_{g}^{2}} .
\end{aligned}
$$

The contributions to $C_{H B}$ depend on different combinations of unknown parameters in the UV theory. They are expected to not be simultaneously tuned to be small in "natural" scenarios. The $C_{H G}$ contribution only proceeds through the scalar quartic interaction. Interestingly, figure $2(\mathrm{a}, \mathrm{b})$ do not vanish in the case of pseudoscalar effective operators; we will return to this point in section 3 .

\subsection{Constraints from Electroweak Precision Data}

In [24], a global fit in the SMEFT has been performed incorporating data from PEP, PETRA, TRISTAN, SpS, Tevatron, SLAC, LEPI and LEPII. Bounds on a number of Wilson coefficients have been obtained and theoretical errors in the SM as well as in the SMEFT have been studied and included, which leads to a relaxation of these bounds. Among these Wilson coefficients one $\left(C_{H W B}\right.$ - also known as the $S$ parameter $)$ is of particular interest as

\footnotetext{
${ }^{6}$ In the full and effective theory, the UV poles cancel, and the IR poles are the same between the two theories by definition. See ref. [23] for more discussion.
} 


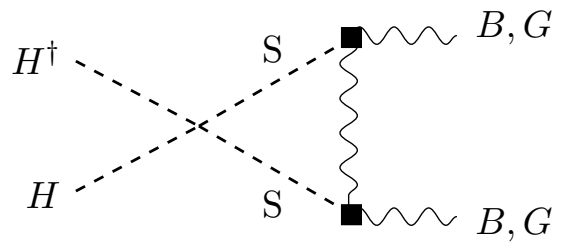

(a)

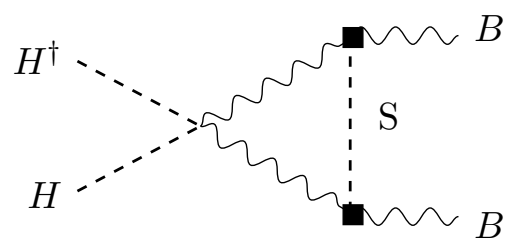

(b)

Figure 2. Direct matching diagrams to $O_{H B}, O_{H G}$ at one loop due to the couplings required for $g g \rightarrow S$ and $S \rightarrow \gamma \gamma$, illustrated with the insertion of a box. Note that these diagrams are also generated by two insertions of the operators with $\tilde{B}_{\mu \nu} B^{\mu \nu}$ and $\tilde{G}_{\mu \nu} G^{\mu \nu}$. These operators are present in the pseudoscalar case. We discuss this case in section 3. Note that a box diagram contribution of this form is not shown as it vanishes due to Lorentz index interchange symmetry.

\begin{tabular}{|c|c|c|c|}
\hline SMEFT error & $0 \%$ & $0.5 \%$ & $1 \%$ \\
\hline$\tilde{C}_{H W B}\left(m_{Z}\right) v^{2} / \Lambda^{2}$ & $-0.0097 \pm 0.018$ & $0.024 \pm 0.028$ & $0.018 \pm 0.030$ \\
\hline$C_{H B}\left(m_{s}\right) v^{2} / \Lambda^{2}$ & $0.12 \pm 0.23$ & $-0.29 \pm 0.35$ & $-0.23 \pm 0.37$ \\
\hline
\end{tabular}

Table 1. Bounds on $\tilde{C}_{H W B}\left(m_{Z}\right) v^{2} / \Lambda^{2}$ and the resulting bounds on $C_{H B}\left(m_{s}\right) v^{2} / \Lambda^{2}$ for a SMEFT error $=\{0 \%, 0.5 \%, 1 \%\}$.

it is generated by $C_{H B}$ by its running from the higher energy scale $\sim m_{s}$ [25-27]. All other Wilson coefficients not generated by the running of $C_{H B}$ and $C_{H G}$ are set to zero in the fit, allowing us using the same data as in [24], to put constraints on $C_{H W B}$ at a low-energy scale $m_{Z}$. This can be translated into bounds on $C_{H B}$ at $m_{s}$ using the RGE for $C_{H W B}$ for which we take $C_{H W B}\left(m_{s}\right) \simeq 0$. The other Wilson coefficients are not exactly zero in any realistic model, but are assumed subdominant. We introduce a theoretical error for the SMEFT to take this into account consistently.

We give the best fit value $\tilde{C}_{H W B}^{\min }\left(m_{Z}\right) \pm \sigma$ with $\tilde{C}_{H W B}=100 C_{H W B}$ as well as resulting bounds on $C_{H B}\left(m_{s}\right)$ for a SMEFT error $=\{0 \%, 0.5 \%, 1 \%\}$ in table 1 . Here the SMEFT error captures the neglect of higher orders (dimension eight operators) and neglected perturbative corrections in fitting the global data in the SMEFT. The remaining errors that enter the analysis are reported directly by the experimental collaborations and specified in detail in ref. [24]. Using the RGE of $C_{H W B}, C_{H B}\left(m_{s}\right)=C_{H B}^{\min }\left(m_{s}\right) \pm \sigma^{\prime}$ with $C_{H B}^{\min }\left(m_{s}\right)=-C_{H W B}^{\min }\left(m_{Z}\right) / K, \sigma^{\prime}=\sigma / K$ and $K$ given by

$$
K=\frac{4 g_{1}^{3} g_{2} y_{h}}{16 \pi^{2}} \log \left[\frac{m_{s}}{m_{Z}}\right] .
$$

Neglecting the running of $C_{H B}$ between the energy scales $m_{s}$ and $m_{h}$, we can use the EWPD to extract bounds on $\kappa_{\gamma}$. We quote the bounds obtained on $\kappa_{\gamma}$ in table 2 which are very weak.

\subsection{Constraints from run I Higgs data}

The operators $O_{H B}$ and $O_{H G}$ map to the $\kappa_{g}$ and $\kappa_{\gamma}$ parameters as

$$
\kappa_{g}=1-\frac{16 \pi^{2} v^{2} C_{H G}\left(m_{h}\right)}{\Lambda^{2} I_{g}}, \quad \kappa_{\gamma}=1-\frac{16 \pi^{2} v^{2} C_{H B}\left(m_{h}\right)}{\Lambda^{2} I_{\gamma}} .
$$




\begin{tabular}{|c|c|c|c|}
\hline SMEFT error & $0 \%$ & $0.5 \%$ & $1 \%$ \\
\hline$\kappa_{\gamma}-1$ & $+12 \pm 23$ & $-29 \pm 34$ & $-22 \pm 37$ \\
\hline
\end{tabular}

Table 2. Bounds on $\kappa_{\gamma}$ from EWPD for a SMEFT error $=\{0 \%, 0.5 \%, 1 \%\}$ in this minimal scenario.

Here we are using notation consistent with ref. [28]. As the $\kappa$ couplings are defined with respect to rescaling the best SM predictions, we retain the NLO QCD correction in the heavy top limit in the expressions $I_{g}, I_{\gamma}$ quoted in ref. [28]. We neglect a correction due to known NLO EW terms that are included in the scaled out SM value experimentally. This introduces an error on the order of $\alpha_{e w} / 4 \pi v^{2} / \Lambda^{2}$. We use $I_{g} \simeq 0.38, I_{\gamma} \simeq-1.6$, retaining only the top quark contribution to the loop functions for the fermions.

In the minimal predictive scenario considered so far, the modified top coupling $\kappa_{t}$ is related to $\kappa_{\gamma}$ and $\kappa_{g}$ as

$$
\kappa_{t}-1=-\frac{v^{2}}{\sqrt{2}}\left(0.46 \frac{\left(\kappa_{g}-1\right) I_{g}}{16 \pi^{2}}+0.0011 \frac{\left(\kappa_{\gamma}-1\right) I_{\gamma}}{16 \pi^{2}}\right) .
$$

so that it is justified to neglect $\kappa_{t}$ as sub-leading and consider the constraints from global Higgs data analyses in just the $\left(\kappa_{g}, \kappa_{\gamma}\right)$ space. So far our discussion has been general.

\subsubsection{Mixing domination}

The tension with the measurements of $\kappa_{g}, \kappa_{\gamma}$ reported for the $125 \mathrm{GeV}$ scalar, when mixing is assumed to dominate the contribution to the low-energy phenomenology through the operator $S H^{\dagger} H$, can be characterized by the parameter $\mathcal{O}$ defined as

$$
\mathcal{O}=\frac{\Delta \sigma(p p \rightarrow S \rightarrow \gamma \gamma)}{8 f b}\left(\frac{\Gamma_{s}}{45 \mathrm{GeV}}\right)\left(\frac{\lambda \Lambda_{c}}{N \times 750 \mathrm{GeV}}\right)^{4}
$$

which is expected to be order one. Here $N$ is a factor for the separation of the cutoff scale and $m_{s}$. By definition $\Lambda_{c} \gtrsim m_{s}$, and we take $N=3$ below. The measured excess leads to the constraint on $\kappa_{g}, \kappa_{\gamma}$

$$
\mathcal{O} \simeq 0.0005\left(\kappa_{\gamma}-1\right)^{2}\left[\left(\kappa_{g}-1\right)^{2}+6.2 \times 10^{-4}\left(\kappa_{\gamma}-1\right)^{2}\right] .
$$

The deviations $\left|\kappa_{g}-1\right|,\left|\kappa_{\gamma}-1\right|$ are constrained to be $\lesssim 0.25$ at $95 \%$ C.L [29]. We illustrate this relation in figure 3. This conflict can be relaxed in a linear fashion if the excess decreases from its reference value of $8 f b$ or the width decreases from its reference value of $45 \mathrm{GeV}$. However, the inconsistency for order one mixing angles is at the level of four orders of magnitude. The coupling of $S$ to $H^{\dagger} H$ that scales as a fourth power must be suppressed from "natural" values to restore consistency with run I data. By the same token, the suppression does not have to be dramatic. An order of magnitude to the fourth power in suppression makes the scenario consistent, considering the experimental uncertainties on the small excess at $750 \mathrm{GeV}$. Two orders of magnitude suppression in the coupling of $S$ to $H^{\dagger} H$ restores good agreement with low-energy Higgs data, and such a suppression is not strongly challenged by naturalness concerns.

Here we are absorbing the dependence on the width into the parameter $\mathcal{O}$ and varying this parameter as in figure 3 in order to not impose a strong preference on the width. 

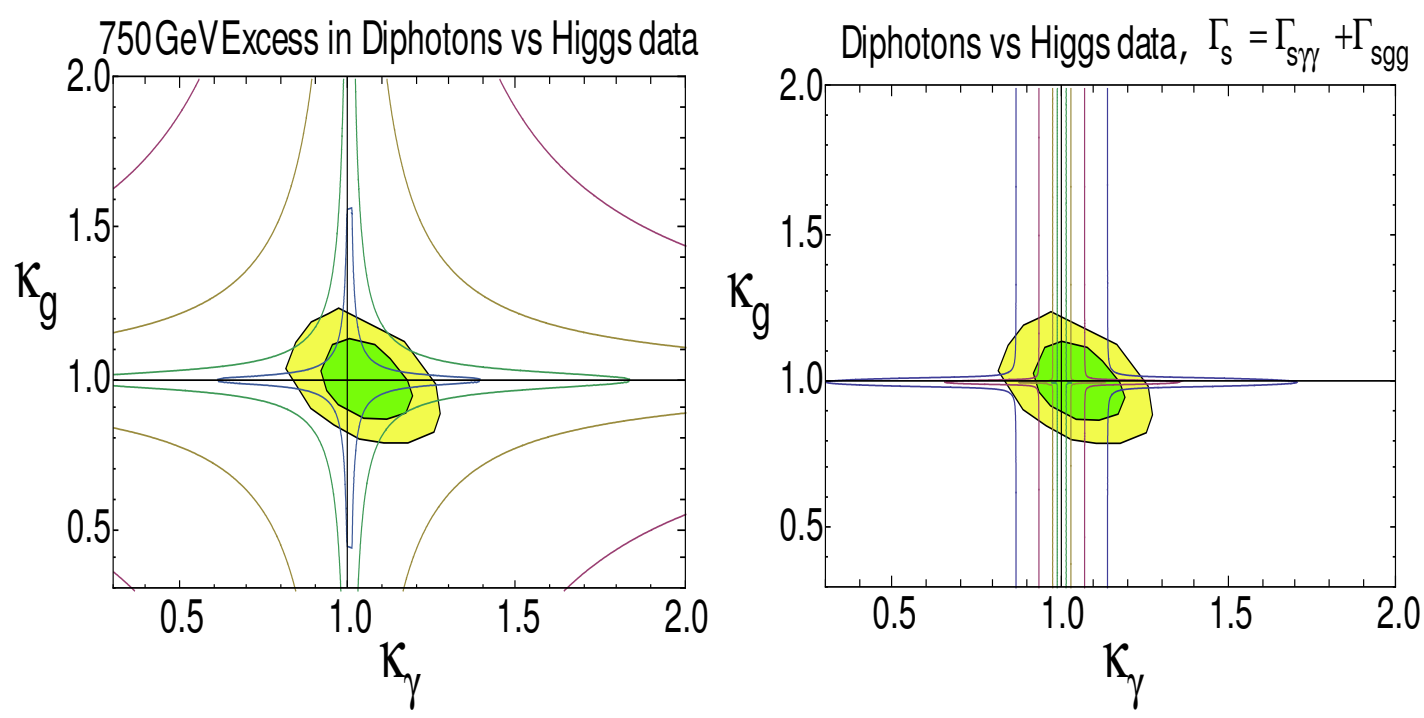

Figure 3. An illustration of the tension between Higgs data and the diphoton excess in minimal scalar models. The curves in the left hand plot are values of $\lambda \Lambda_{c} / N \times 750 \mathrm{GeV}=$ $\{0.1,0.05,0.02,0.01\}$ coming in from the outermost curve in eq. (2.31). The right hand plot shows $\lambda \Lambda_{c} / N \times 750 \mathrm{GeV}=\{0.1,0.05,0.02,0.01\}$ in the case that the width is reproduced by just the gluonic and photon production and decay, given by eq. (2.32). In both figures, the curves are overlaid on the $68 \%$ and $95 \%$ CL curves from the run I Atlas-CMS Higgs combination, fitting only to $\kappa_{g}, \kappa_{\gamma}[29]$.

\subsubsection{Reproducing the width}

If we further fix the condition that $\Gamma_{s}=\Gamma(s \rightarrow \gamma \gamma)+\Gamma(s \rightarrow g g)$, we derive the constraint equation to reproduce the excess

$$
\frac{\Delta \sigma(p p \rightarrow S \rightarrow \gamma \gamma)}{8 f b}\left(\frac{\lambda \Lambda_{c}}{N m_{s}}\right)^{2}=0.06 N^{2}\left(\kappa_{\gamma}-1\right)^{2} \frac{\left(\kappa_{g}-1\right)^{2}+6.2 \times 10^{-4}\left(\kappa_{\gamma}-1\right)^{2}}{\left(\kappa_{g}-1\right)^{2}+0.017\left(\kappa_{\gamma}-1\right)^{2}}
$$

The effects of this condition are shown in figure 3. Note that reducing the width in this case quickly allows consistency with lower energy data, by making the coupling required to reproduce the excess smaller. Here we are absorbing the dependence on the width into the parameter $\mathcal{O}$ and varying this parameter as in figure 3 in order to not impose a strong preference on the width. We are only enforcing that the width as it varies is reproduced by the production and decay through $\gamma \gamma$ and $g g$.

\subsubsection{Matching domination}

As we have stressed, the Wilson coefficients $C_{H B}$ and $C_{H G}$ also receive contributions independent of the mixing angle. As these matching coefficients are generated by loops involving two insertions of the new scalar's coupling to SM field strengths, they lead to a relation between the measured excess and Higgs data which scales as just a square rather than a fourth power. 
In the limit where these matching contributions are the only contribution to the shifts in the Higgs couplings and $\lambda_{3} \gg \frac{y_{h}^{2} g_{1}^{2}}{2}$, we can express the signal rate as

$$
\frac{\Delta \sigma(p p \rightarrow S \rightarrow \gamma \gamma)}{8 f b}\left(\frac{\Gamma_{s}}{45 \mathrm{GeV}}\right)=\frac{1.7 \times 10^{5}}{\lambda_{3}^{2}}\left(\kappa_{\gamma}-1\right)\left(\left(\kappa_{g}-1\right)+2.2 \times 10^{-2}\left(\kappa_{\gamma}-1\right)\right) .
$$

These matching contributions to the Higgs observables are not significantly constrained by the run I Higgs data.

\section{Consistency of pseudoscalar models with lower energy data}

A $J^{P}=0^{-}$pseudoscalar boson interpretation of the $S$ particle related to the diphoton excess would not lead to direct mixing with the $J^{P}=0^{+}$Higgs boson. This further protects this model from related low-energy phenomenology constraints. At the one-loop level such interactions still generate $H^{\dagger} H B^{\mu \nu} B_{\mu \nu}$ through the diagrams shown in figure 2. We have calculated these contributions and found them to be identical to the scalar case; therefore, the discussion in section 2.5.3 applies in full to pseudoscalar models. This leaves models which employ a fundamental pseudoscalar to explain the diphoton excess largely unconstrained.

However, the constraints on mixing discussed above still apply to a heavy sector with such a state, which generally arise when the pseudoscalar being considered is a bound state of new strong dynamics. To elaborate on this point concretely, we utilize the models discussed in ref. [14]. Consider a minimal "hidden pion" model of a pseudoscalar given in ref. [14], which also introduces heavy vector-like hidden quarks at the scale $\Lambda_{c}$, and a new $\mathrm{SU}(N)$ gauge group. This leads to the effective interactions of the "hidden pion" $\phi$

$$
\mathcal{L}_{\phi}=c_{\tilde{G}}^{\phi} \frac{\phi}{f} G^{\mu \nu} \tilde{G}_{\mu \nu}+c_{\tilde{B}}^{\phi} \frac{\phi}{f} B^{\mu \nu} \tilde{B}_{\mu \nu}
$$

with $\tilde{G}_{\mu \nu}=\frac{1}{2} \epsilon_{\mu \nu \sigma \rho} G^{\sigma \rho}$, and for this model

$$
c_{\tilde{G}}^{\phi}=-\frac{N g_{3}^{2}}{32 \sqrt{6} \pi^{2}}, \quad c_{\tilde{B}}^{\phi}=-\frac{9\left(a^{2}-b^{2}\right) N g_{1}^{2}}{80 \sqrt{6} \pi^{2}},
$$

where $a, b$ are the hypercharges of the constituent particles. In this case the decay to diphotons is considered as analogous to that of the neutral pion, where the decay is calculable and due to the chiral anomaly. Accompanying new scalar mesons of the new confining interactions are generically expected. These scalars will be bounded by the mixing constraints determined in the previous sections. The QCD example is the very wide $\sigma$ meson, which decays dominantly as $\sigma \rightarrow \pi \pi$, but does have a known decay into $\gamma \gamma$. We can develop a very rough understanding of the relationship between the couplings of a composite pseudoscalar to photons and those of a corresponding scalar on the basis of the constituent dynamics; one expects that the corresponding couplings are related by

$$
\frac{c_{\tilde{B}}^{S}}{c_{\tilde{B}}^{\phi}} \sim \frac{a^{2}+b^{2}}{a^{2}-b^{2}} \frac{\Psi^{s}(0)}{\Psi^{\phi}(0)}
$$




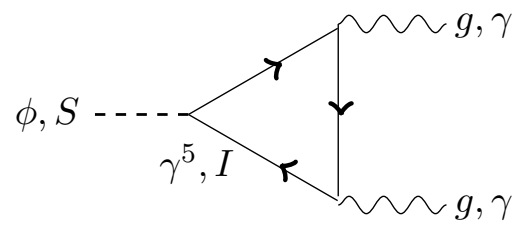

Figure 4. Anomaly diagrams for $\phi$ and $s$.

here $\Psi^{s}(0)$ and $\Psi^{\phi}(0)$ are the wavefunctions at the origin of the bound states. This is expected if the constituents mediating the coupling to two photons are identical, leading to the same loop function for two mesons. This corresponds to considering a scalar with identical flavor quantum numbers as the pseudoscalar. In the pseudoscalar case there is an insertion of $\gamma^{5}$ in the diagram leading to the difference between squared hypercharges, while in the scalar case a unit matrix sums the squared charges, see figure 4.

There is no reason to expect $\Psi^{s}(0) \ll \Psi^{\phi}(0)$ in general. The same reasoning applies to decays to $g g$. Although $m_{s}$ can exceed $m_{\phi}$, the typical separation expected is $m_{s} / m_{\phi} \lesssim 4 \pi$. The mixing bounds of eq. (2.31) and eq. (2.32) then apply to the new scalar. To determine the bounds in detail requires a model dependent matching calculation to fix $c_{\tilde{B}}^{S}, c_{\tilde{G}}^{S}$.

\section{Conclusions}

We have examined the consistency of run I Higgs data and a putative diphoton excess at $750 \mathrm{GeV}$, considering scalar and pseudoscalar states that have an impact on lower energy phenomenology using the SMEFT formalism. We find that large mixings of a $750 \mathrm{GeV}$ state (i.e. Wilson coefficients of the relevant operator $S H^{\dagger} H$ proximate to the cutoff scale) are challenged by these concerns, and have examined the corresponding naturalness bounds on the radiatively generated Wilson coefficient, due to the interactions required to produce the excess in diphotons. In general, we find that once a loop suppression of this Wilson coefficient is introduced, scalar models can be viable, and pseudoscalar models are more protected from dangerous low-energy effects. One-loop matchings due to the pseudoscalar interactions do generate the operator $O_{H B}=H^{\dagger} H B^{\mu \nu} B_{\mu \nu}$. The diphoton excess is not strongly challenged by consistency with lower energy data we have considered, in the simple scenarios we have examined.

\section{Acknowledgments}

JC is grateful to the NBIA for its generous hospitality during this work, which is also supported by NSERC (Canada). MT and WS acknowledge generous support from the Villum Fonden. We thank Angelo Monteaux for a helpful comment, and Zuowei Liu for useful discussions.

Note added. As well as the papers cited in the text, and utilized in this work, the following papers appeared on the archive discussing the $750 \mathrm{GeV}$ excess prior to this paper [30-62]. 


\section{A One-loop results}

Figure 2(a) gives the one-loop contribution to the Wilson coefficient $C_{H B}$ matching condition

$$
\frac{\Delta^{a} C_{H B}\left(m_{s}\right) g_{1}^{2}}{\Lambda^{2}}=\frac{\lambda_{3}}{4 \pi^{2}}\left[\frac{5}{2}-\frac{\pi}{\sqrt{3}}\right] \frac{g_{1}^{4} c_{B}^{2}}{\Lambda_{\gamma}^{2}},
$$

while figure $2(\mathrm{~b})$ gives the contribution

$$
\frac{\Delta^{a} C_{H B}\left(m_{s}\right) g_{1}^{2}}{\Lambda^{2}}=\frac{1}{4 \pi^{2}}\left[-\frac{5}{2}+\frac{\pi^{2}}{12}\right] \frac{g_{1}^{6} y_{h}^{2} c_{B}^{2}}{\Lambda_{\gamma}^{2}},
$$

when calculating the unbroken phase of $\mathrm{SU}(2)_{\mathrm{L}} \times \mathrm{U}(1)_{\mathrm{Y}}$ to simplify the matching. Note we take the real part of the amplitude in the matching as the Wilson coefficient of the Hermitian operators are real. Figure 2(b) vanishes for $C_{H G}$ while figure 1(a) is the obvious modification of the quoted result for this operator.

Open Access. This article is distributed under the terms of the Creative Commons Attribution License (CC-BY 4.0), which permits any use, distribution and reproduction in any medium, provided the original author(s) and source are credited.

\section{References}

[1] ATLAS collaboration, Search for resonances decaying to photon pairs in 3.2fb-1 of $\mathrm{pp}$ collisions at $\sqrt{s}=13 \mathrm{TeV}$ with the ATLAS detector, ATLAS-CONF-2015-081 (2015).

[2] CMS collaboration, Search for new physics in high mass diphoton events in proton-proton collisions at $\sqrt{s}=13 \mathrm{TeV}$, CMS-PAS-EXO-15-004 (2015).

[3] K. Cranmer, Testing look-elsewhere effect from combining two searches for the same particle using Gaussian Processes, https://github.com/cranmer/look-elsewhere-2d/blob/master/ two-experiment-lee.ipynb.

[4] L.D. Landau, On the angular momentum of a s cystem of two photons, Dokl. Akad. Nauk Ser. Fiz. 60 (1948) 207.

[5] C.-N. Yang, Selection Rules for the Dematerialization of a Particle Into Two Photons, Phys. Rev. 77 (1950) 242 [INSPIRE].

[6] C. Csáki, J. Hubisz and J. Terning, Minimal model of a diphoton resonance: Production without gluon couplings, Phys. Rev. D 93 (2016) 035002 [arXiv:1512.05776] [INSPIRE].

[7] S. Fichet, G. von Gersdorff and C. Royon, Scattering Light by Light at $750 \mathrm{GeV}$ at the LHC, arXiv: 1512.05751 [INSPIRE].

[8] S. Fichet, G. von Gersdorff and C. Royon, Measuring the diphoton coupling of a $750 \mathrm{GeV}$ resonance, arXiv: 1601.01712 [INSPIRE].

[9] C. Csáki, J. Hubisz, S. Lombardo and J. Terning, Gluon vs. Photon Production of a $750 \mathrm{GeV}$ Diphoton Resonance, arXiv:1601.00638 [INSPIRE].

[10] R. Franceschini et al., What is the $\gamma \gamma$ resonance at 750 GeV?, JHEP 03 (2016) 144 [arXiv: 1512.04933] [INSPIRE]. 
[11] A.D. Martin, W.J. Stirling, R.S. Thorne and G. Watt, Parton distributions for the LHC, Eur. Phys. J. C 63 (2009) 189 [arXiv:0901.0002] [inSPIRE].

[12] D. Aloni, K. Blum, A. Dery, A. Efrati and Y. Nir, On a possible large width $750 \mathrm{GeV}$ diphoton resonance at ATLAS and CMS, arXiv:1512.05778 [INSPIRE].

[13] ATLAS collaboration, Search for new resonances in $W \gamma$ and $Z \gamma$ final states in pp collisions at $\sqrt{s}=8 \mathrm{TeV}$ with the ATLAS detector, Phys. Lett. B 738 (2014) 428 [arXiv:1407.8150] [INSPIRE].

[14] K. Harigaya and Y. Nomura, Composite Models for the 750 GeV Diphoton Excess, Phys. Lett. B 754 (2016) 151 [arXiv:1512.04850] [INSPIRE].

[15] E.E. Jenkins, A.V. Manohar and M. Trott, Renormalization Group Evolution of the Standard Model Dimension Six Operators I: Formalism and lambda Dependence, JHEP 10 (2013) 087 [arXiv: 1308.2627] [INSPIRE].

[16] E.E. Jenkins, A.V. Manohar and M. Trott, Renormalization Group Evolution of the Standard Model Dimension Six Operators II: Yukawa Dependence, JHEP 01 (2014) 035 [arXiv: 1310.4838] [INSPIRE].

[17] R. Alonso, E.E. Jenkins, A.V. Manohar and M. Trott, Renormalization Group Evolution of the Standard Model Dimension Six Operators III: Gauge Coupling Dependence and Phenomenology, JHEP 04 (2014) 159 [arXiv:1312.2014] [INSPIRE].

[18] R. Alonso, H.-M. Chang, E.E. Jenkins, A.V. Manohar and B. Shotwell, Renormalization group evolution of dimension-six baryon number violating operators, Phys. Lett. B 734 (2014) 302 [arXiv:1405.0486] [INSPIRE].

[19] B. Grzadkowski, M. Iskrzynski, M. Misiak and J. Rosiek, Dimension-Six Terms in the Standard Model Lagrangian, JHEP 10 (2010) 085 [arXiv: 1008.4884] [INSPIRE].

[20] R. Contino, M. Ghezzi, C. Grojean, M. Muhlleitner and M. Spira, Effective Lagrangian for a light Higgs-like scalar, JHEP 07 (2013) 035 [arXiv: 1303.3876] [INSPIRE].

[21] A. Falkowski, O. Slone and T. Volansky, Phenomenology of a $750 \mathrm{GeV}$ Singlet, JHEP 02 (2016) 152 [arXiv: 1512.05777] [INSPIRE].

[22] A. Manohar and H. Georgi, Chiral Quarks and the Nonrelativistic Quark Model, Nucl. Phys. B 234 (1984) 189 [INSPIRE].

[23] A.V. Manohar, Deep inelastic scattering as $x \rightarrow 1$ using soft collinear effective theory, Phys. Rev. D 68 (2003) 114019 [hep-ph/0309176] [INSPIRE].

[24] L. Berthier and M. Trott, Consistent constraints on the Standard Model Effective Field Theory, JHEP 02 (2016) 069 [arXiv: 1508.05060] [INSPIRE].

[25] K. Hagiwara, S. Ishihara, R. Szalapski and D. Zeppenfeld, Low-energy constraints on electroweak three gauge boson couplings, Phys. Lett. B 283 (1992) 353 [INSPIRE].

[26] S. Alam, S. Dawson and R. Szalapski, Low-energy constraints on new physics revisited, Phys. Rev. D 57 (1998) 1577 [hep-ph/9706542] [InSPIRE].

[27] C. Grojean, E.E. Jenkins, A.V. Manohar and M. Trott, Renormalization Group Scaling of Higgs Operators and $h \rightarrow \gamma \gamma$ Decay, JHEP 04 (2013) 016 [arXiv: 1301.2588] [INSPIRE].

[28] A.V. Manohar and M.B. Wise, Modifications to the properties of the Higgs boson, Phys. Lett. B 636 (2006) 107 [hep-ph/0601212] [INSPIRE]. 
[29] ATLAS and CMS collaborations, Measurements of the Higgs boson production and decay rates and constraints on its couplings from a combined ATLAS and CMS analysis of the LHC pp collision data at $\sqrt{s}=7$ and 8 TeV, ATLAS-CONF-2015-044 (2015).

[30] A. Alves, A.G. Dias and K. Sinha, The $750 \mathrm{GeV}$ S-cion: Where else should we look for it?, arXiv: 1512.06091 [INSPIRE].

[31] Y. Bai, J. Berger and R. Lu, A 750 GeV Dark Pion: Cousin of a Dark G-parity-odd WIMP, arXiv: 1512.05779 [INSPIRE].

[32] P. Agrawal, J. Fan, B. Heidenreich, M. Reece and M. Strassler, Experimental Considerations Motivated by the Diphoton Excess at the LHC, arXiv:1512.05775 [INSPIRE].

[33] A. Ahmed, B.M. Dillon, B. Grzadkowski, J.F. Gunion and Y. Jiang, Higgs-radion interpretation of $750 \mathrm{GeV}$ di-photon excess at the LHC, arXiv: 1512.05771 [INSPIRE].

[34] J. Chakrabortty, A. Choudhury, P. Ghosh, S. Mondal and T. Srivastava, Di-photon resonance around $750 \mathrm{GeV}$ : shedding light on the theory underneath, arXiv:1512.05767 [INSPIRE].

[35] L. Bian, N. Chen, D. Liu and J. Shu, A hidden confining world on the $750 \mathrm{GeV}$ diphoton excess, arXiv: 1512.05759 [INSPIRE].

[36] D. Curtin and C.B. Verhaaren, Quirky Explanations for the Diphoton Excess, Phys. Rev. D 93 (2016) 055011 [arXiv: 1512.05753] [INSPIRE].

[37] W. Chao, R. Huo and J.-H. Yu, The Minimal Scalar-Stealth Top Interpretation of the Diphoton Excess, arXiv:1512.05738 [INSPIRE].

[38] S.V. Demidov and D.S. Gorbunov, On sgoldstino interpretation of the diphoton excess, arXiv: 1512.05723 [INSPIRE].

[39] J.M. No, V. Sanz and J. Setford, See-Saw Composite Higgses at the LHC: Linking Naturalness to the $750 \mathrm{GeV}$ Di-Photon Resonance, arXiv:1512.05700 [INSPIRE].

[40] D. Becirevic, E. Bertuzzo, O. Sumensari and R.Z. Funchal, Can the new resonance at LHC be a CP-Odd Higgs boson?, arXiv:1512.05623 [INSPIRE].

[41] P. Cox, A.D. Medina, T.S. Ray and A. Spray, Diphoton Excess at $750 \mathrm{GeV}$ from a Radion in the Bulk-Higgs Scenario, arXiv:1512.05618 [INSPIRE].

[42] R. Martinez, F. Ochoa and C.F. Sierra, Diphoton decay for a $750 \mathrm{GeV}$ scalar boson in an $\mathrm{U}(1)^{\prime}$ model, arXiv: 1512.05617 [INSPIRE].

[43] A. Kobakhidze, F. Wang, L. Wu, J.M. Yang and M. Zhang, $750 \mathrm{GeV}$ diphoton resonance in a top and bottom seesaw model, arXiv:1512.05585 [INSPIRE].

[44] S. Matsuzaki and K. Yamawaki, $750 \mathrm{GeV}$ Diphoton Signal from One-Family Walking Technipion, arXiv:1512.05564 [INSPIRE].

[45] Q.-H. Cao, Y. Liu, K.-P. Xie, B. Yan and D.-M. Zhang, A Boost Test of Anomalous Diphoton Resonance at the LHC, arXiv:1512.05542 [INSPIRE].

[46] B. Dutta, Y. Gao, T. Ghosh, I. Gogoladze and T. Li, Interpretation of the diphoton excess at CMS and ATLAS, Phys. Rev. D 93 (2016) 055032 [arXiv:1512.05439] [InSPIRE].

[47] C. Petersson and R. Torre, The $750 \mathrm{GeV}$ diphoton excess from the goldstino superpartner, arXiv:1512.05333 [INSPIRE].

[48] E. Molinaro, F. Sannino and N. Vignaroli, Minimal Composite Dynamics versus Axion Origin of the Diphoton excess, arXiv:1512.05334 [INSPIRE]. 
[49] R.S. Gupta, S. Jäger, Y. Kats, G. Perez and E. Stamou, Interpreting a $750 \mathrm{GeV}$ Diphoton Resonance, arXiv:1512.05332 [INSPIRE].

[50] B. Bellazzini, R. Franceschini, F. Sala and J. Serra, Goldstones in Diphotons, arXiv: 1512.05330 [INSPIRE].

[51] M. Low, A. Tesi and L.-T. Wang, A pseudoscalar decaying to photon pairs in the early LHC Run 2 data, JHEP 03 (2016) 108 [arXiv: 1512.05328] [INSPIRE].

[52] J. Ellis, S.A.R. Ellis, J. Quevillon, V. Sanz and T. You, On the Interpretation of a Possible $\sim 750 \mathrm{GeV}$ Particle Decaying into $\gamma \gamma$, arXiv:1512.05327 [INSPIRE].

[53] S.D. McDermott, P. Meade and H. Ramani, Singlet Scalar Resonances and the Diphoton Excess, Phys. Lett. B 755 (2016) 353 [arXiv:1512.05326] [INSPIRE].

[54] T. Higaki, K.S. Jeong, N. Kitajima and F. Takahashi, The QCD Axion from Aligned Axions and Diphoton Excess, Phys. Lett. B 755 (2016) 13 [arXiv:1512.05295] [INSPIRE].

[55] S. Di Chiara, L. Marzola and M. Raidal, First interpretation of the 750 GeV di-photon resonance at the $L H C$, arXiv:1512.04939 [INSPIRE].

[56] A. Pilaftsis, Diphoton Signatures from Heavy Axion Decays at the CERN Large Hadron Collider, Phys. Rev. D 93 (2016) 015017 [arXiv:1512.04931] [INSPIRE].

[57] D. Buttazzo, A. Greljo and D. Marzocca, Knocking on new physics' door with a scalar resonance, Eur. Phys. J. C 76 (2016) 116 [arXiv:1512.04929] [INSPIRE].

[58] S. Knapen, T. Melia, M. Papucci and K. Zurek, Rays of light from the LHC, arXiv: 1512.04928 [INSPIRE].

[59] Y. Nakai, R. Sato and K. Tobioka, Footprints of New Strong Dynamics via Anomaly, arXiv: 1512.04924 [INSPIRE].

[60] A. Angelescu, A. Djouadi and G. Moreau, Scenarii for interpretations of the LHC diphoton excess: two Higgs doublets and vector-like quarks and leptons, Phys. Lett. B 756 (2016) 126 [arXiv: 1512.04921] [INSPIRE].

[61] M. Backovic, A. Mariotti and D. Redigolo, Di-photon excess illuminates Dark Matter, JHEP 03 (2016) 157 [arXiv: 1512.04917] [INSPIRE].

[62] Y. Mambrini, G. Arcadi and A. Djouadi, The LHC diphoton resonance and dark matter, Phys. Lett. B 755 (2016) 426 [arXiv: 1512.04913] [INSPIRE]. 